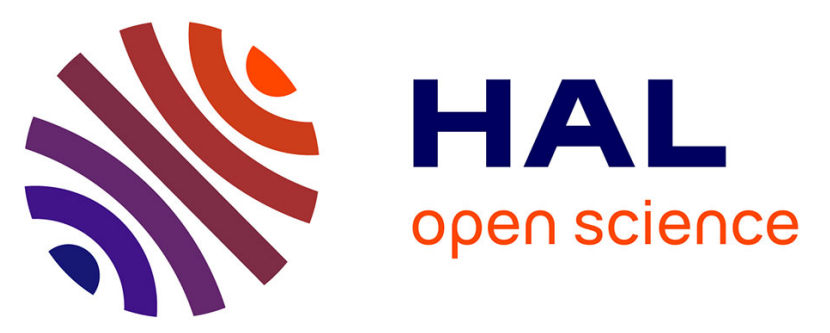

\title{
Relatório 05 - Por que é inadequado divulgar os indicadores de mortes por data de óbito? Uma explicação das diferentes metodologias para quantificar os óbitos durante a epidemia
}

Americo Cunha Jr, Lisandro Lovisolo, Malú Grave, Rodrigo Burgos, Adriano Cortês, Karla Figueiredo, Roberto Velho, Bruna Silveira Pavlack, Diego H S Catalão, Diego Matos, et al.

\section{- To cite this version:}

Americo Cunha Jr, Lisandro Lovisolo, Malú Grave, Rodrigo Burgos, Adriano Cortês, et al.. Relatório 05 - Por que é inadequado divulgar os indicadores de mortes por data de óbito? Uma explicação das diferentes metodologias para quantificar os óbitos durante a epidemia. [Technical Report] COVID-19: Observatório Fluminense. 2020. hal-02887105

\section{HAL Id: hal-02887105 https://hal.science/hal-02887105}

Submitted on 2 Jul 2020

HAL is a multi-disciplinary open access archive for the deposit and dissemination of scientific research documents, whether they are published or not. The documents may come from teaching and research institutions in France or abroad, or from public or private research centers.
L'archive ouverte pluridisciplinaire HAL, est destinée au dépôt et à la diffusion de documents scientifiques de niveau recherche, publiés ou non, émanant des établissements d'enseignement et de recherche français ou étrangers, des laboratoires publics ou privés.

\section{(ㅇ)(1) $\$$}

Distributed under a Creative Commons Attribution - NonCommercial - NoDerivatives 44.0 


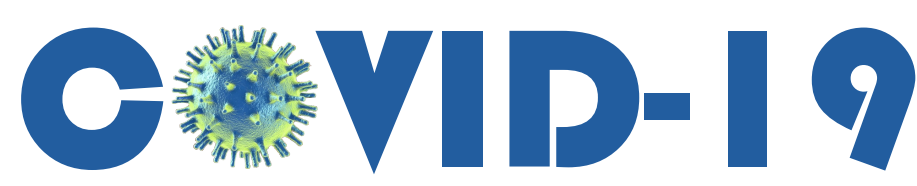 OBSERVATÓRIO FLUMINENSE \\ www.covid19rj.org}

\section{Relatório 05 \\ Por que é inadequado divulgar os indicadores de mortes por data de óbito? Uma explicação das diferentes metodologias para quantificar os óbitos durante a epidemia}

Americo Cunha Jr*, Lisandro Lovisolo*, Malú Grave*, Rodrigo Burgos*, Adriano Cortês, Karla Figueiredo, Roberto Velho, Bruna Pavlack, Diego H. S. Catalão, Diego Matos, Eber Dantas, Gabrielle Pereira, João

Pedro Norenberg, Julio Basilio, Leonardo de la Roca, Lucas Chaves, Luiz F. S. Coelho, Marcos Issa, Michel Tosin, Roberto Luo, Amanda Cunha Guyt, Luthiana Soares

*Contribuíram igualmente para elaboração deste relatório.

Os autores declaram nenhum conflito de interesse.

Correspondência: americo@ime.uerj.br, lisandro@uerj.br, rburgos@eng.uerj.br

Rio de Janeiro, 19 de junho de 2020

Sugestão de citação:

A. Cunha Jr, et al. Relatório 05 Por que é inadequado divulgar os indicadores de mortes por data de óbito?: Uma explicação das diferentes metodologias para quantificar os óbitos durante a epidemia. COVID-19: Observatório Fluminense (19/06/2020), DOI:

https://doi.org/10.12957/eduerj.covid19rj.relatorio5 


\section{COVID-19: Observatório Fluminense}

Esta é uma inciativa independente de pesquisadores, que congrega uma equipe multidisciplinar (matemática, engenharias, computação, arquitetura, jornalismo), para responder algumas demandas emergentes com o avanço da pandemia de COVID-19 em âmbito nacional. O interesse individual dos membros da equipe pela pandemia levou, naturalmente, ao intercâmbio de informações entre pesquisadores e estudantes, tais como fontes de dados, análises gráficas, notícias, relatórios e artigos científicos e, especialmente, ferramentas matemáticas empregadas na modelagem e análise do progresso de epidemias. Essa interação resultou na organização de uma força tarefa para buscar algumas soluções em termos de análise e visualização de dados, modelagem matemática da epidemia, bem como para produzir material educacional para estudantes interessados no tema e para o público em geral.

Dessa forma, os objetivos da presente iniciativa se articulam em tornos dos seguintes tópicos:

- Monitorar, em âmbito nacional e no estado do Rio de Janeiro, o progresso da pandemia de COVID-19;

- Construir gráficos e outras entidades para visualização de dados que permitam acompanhar e analisar o progresso da pandemia de modo claro e pedagógico;

- Fazer previsões confiáveis sobre o progresso de curto prazo da pandemia (número de infectados, número de óbitos, variações dos mesmos etc);

- Desenvolver material educativo de alto nível na área de modelagem matemática de epidemias;

- Desenvolver e divulgar material informativo de qualidade para o público interessado.

Mais informações sobre a iniciativa podem ser obtidas em www.covid19rj.org. Todos os gráficos e informações apresentados neste relatório, bem como o mesmo demais documentos produzidos pela equipe COVID19RJ, podem ser encontrados no repositório https://github.com/americocunhajr/COVID19RJ.

Outras informações e resultados relevantes também podem ser vistos nas redes sociais da iniciativa:

wWW. instagram.com/portalcovid19rj

WWW.facebook.com/portalcovid19rj

WW. twitter.com/portalcovid19rj 


\section{Equipe de trabalho}

\section{Professores / Pesquisadores:}

Adriano Cortês

(UFRJ)

Americo Cunha

(UERJ)

(UERJ)

(UERJ)

Lisandro Lovisolo

Malú Grave

(UFRJ)

(UFRGS)

Roberto M. Velho

Rodrigo Burgos

\section{Estudantes:}

Bruna Pavlack

Diego H.S. Catalão

Diego Matos

Eber Dantas

Gabrielle Pereira

João P. Norenberg

Julio Basilio

Leonardo de la Roca

Lucas Chaves

Luiz F. S. Coelho

Marcos Issa

Michel Tosin

Roberto Luo

\section{Design Gráfico:}

Amanda Cunha Guyt

(CCSF)

(IFMS)

(UFF)

(UERJ)

(UFRJ)

(UERJ)

(UNESP)

(UERJ)

(UERJ)

(UFU)

(UERJ)

(UERJ)

(UERJ)

(UERJ)

\section{Comunicação:}

Luthiana Soares adriano@caxias.ufrj.br

americo@ime.uerj.br

karla.figueiredo@gmail.com

lisandro@uerj.br

malugrave@nacad.ufrj.br

roberto.velho@gmail.com

rburgos@eng.uerj.br

bruna.pavlack@ifms.edu.br

diegocatalao@id.uff.br

diego.matos@uerj.br

eberdantas@ufrj.br

pereira.gabrielle@graduacao.uerj.br

p.norenberg@unesp.br

basilio.julio@posgraduacao.uerj.br

delaroca@protonmail.com

Lucasfernando@ufu.br

Ifscoelho@ieee.org

marcos.issa@uerj.br

michel.tosin@uerj.br

cai.roberto@graduacao.uerj.br

aguyt@mail.ccsf.edu

luthianassoares@gmail.com 


\section{Fontes de dados}

Os dados de óbitos exibidos empregados neste relatório advêm do painel de monitoramento da epidemia mantido pela Secretaria de Saúde do Estado do Rio de Janeiro obtidos nos dias 26/05 e 09/06 [1]:

http://painel.saude.rj.gov.br

\section{Contestação de Responsabilidade}

Os resultados apresentados neste relatório resultam de simulações computacionais e análises estatísticas conduzidas com auxílio de diversos tipos de modelo matemático, que utilizam informações de várias bases de dados. A qualidade dos resultados e confiança nos valores apresentados deriva diretamente da qualidade, completude, consistência, e acurácia das fontes empregadas. Assim sendo, eventuais erros e imprecisões podem ocorrer nas análises, independentemente dos rigores técnico-científico e ético seguidos pela equipe COVID-19: Observatório Fluminense. 


\section{As metodologias de divulgação dos óbitos}

Recentemente, o Ministério da Saúde e algumas secretarias estaduais de saúde cogitaram adotar uma nova metodologia de divulgação dos dados referentes à COVID-19. O Ministério da Saúde propôs divulgar somente a informação dos óbitos ocorridos e confirmados na data de divulgação de dados e não os registrados naquele dia, como é a praxe atual. Inclusive, todos os relatórios de acompanhamento produzidos por esta inciativa divulgam os dados desta forma [2, 3, 4, 5].

Além disso, algumas secretarias passaram a apresentar gráficos apenas com os óbitos em que se conhece a data do falecimento em vez de considerar todos os registros diários. A seguir serão explicadas as diferenças entre as duas metodologias de quantificação de óbitos devidos à COVID-19 e as implicações que uma eventual mudança na apresentação dos dados poderia trazer para o acompanhamento do progresso da epidemia e decisões sobre como proteger a população.

\subsection{Qual a diferença entre as duas metodologias?}

A Figura 1 busca esclarecer a diferença entre as duas metodologias (divulgação dos dados pela data de óbito $\times$ pela data de registro). A curva vermelha representa os óbitos divulgados no boletim de $26 / 05$, na qual o valor vertical se refere à quantidade real de óbitos ocorridos na data do eixo horizontal sabidos até 26/05. A curva amarela apresenta o mesmo tipo de curva (óbitos $\times$ data) porém conforme conhecidos até o dia 09/06. Já, as barras cinzas apresentam os óbitos conforme suas datas de registro e divulgação pela secretaria estadual de saúde do RJ. Cumpre observar que nesse número há óbitos de diversos dias.

Nota-se que a curva vermelha é um retrato parcial dos óbitos até o dia 26/05, representando apenas óbitos com data de falecimento conhecida até o dia 26/05. No dia 09/06, já se tinha mais informação sobre os óbitos ocorridos naquele período (até 26/05) e consequentemente o número de óbitos com data de falecimento conhecida aumentou consideravelmente (curva amarela). O mesmo raciocínio é aplicável à curva do dia 09/06; provavelmente, no dia 09/06 ainda não se conheciam todos os óbitos ocorridos até a data e assim a curva de óbitos diários até essa data deverá aumentar nas próximas divulgações.

Percebe-se, que o emprego de curvas com dados parciais poderá apresentar uma falsa tendência de queda na quantidade de mortes diárias se considerarmos os dias anteriores e próximos à data de divulgação. Isso decorre da existência de mortes ainda não notificadas por conta de atrasos na confirmação da causa da morte, seu registro e divulgação da mesma. Este é o principal motivo da inadequação da forma de apresentação dos dados por data de óbito, pois poderia acarretar uma falsa impressão de decréscimo na curva de número de mortes diárias. Essa aparente queda é apenas um problema de desconhecimento da totalidade dos óbitos ocorridos naqueles dias por quem divulga os dados. Muitas vezes, a informação de um óbito demora dias para chegar até a contabilização das secretarias de saúde e esse atraso pode fazer com que os dados relatados e divulgados em um dia específico não representem a totalidade de óbitos do mesmo.

A curva referente ao dia 09/06 permite concluir claramente que o número de pessoas que realmente morreram no dia 26/05 é muito maior do que os conhecidos no dia 26/05. Inclusive, podemos conjecturar com grande probabilidade de acerto que esse número ainda aumentará em divulgações posteriores.Pelo gráfico fica claro que ambas as curvas de divulgação por data de óbito (vermelha e amarela) apresentam uma aparente tendência de queda, quando na verdade a tendência de alta das barras cinzas (de dados divulgados no dia do registro do óbito, independentemente da data real de óbito) é a que melhor representa a situação que ocorre em cada dia de divulgação. 


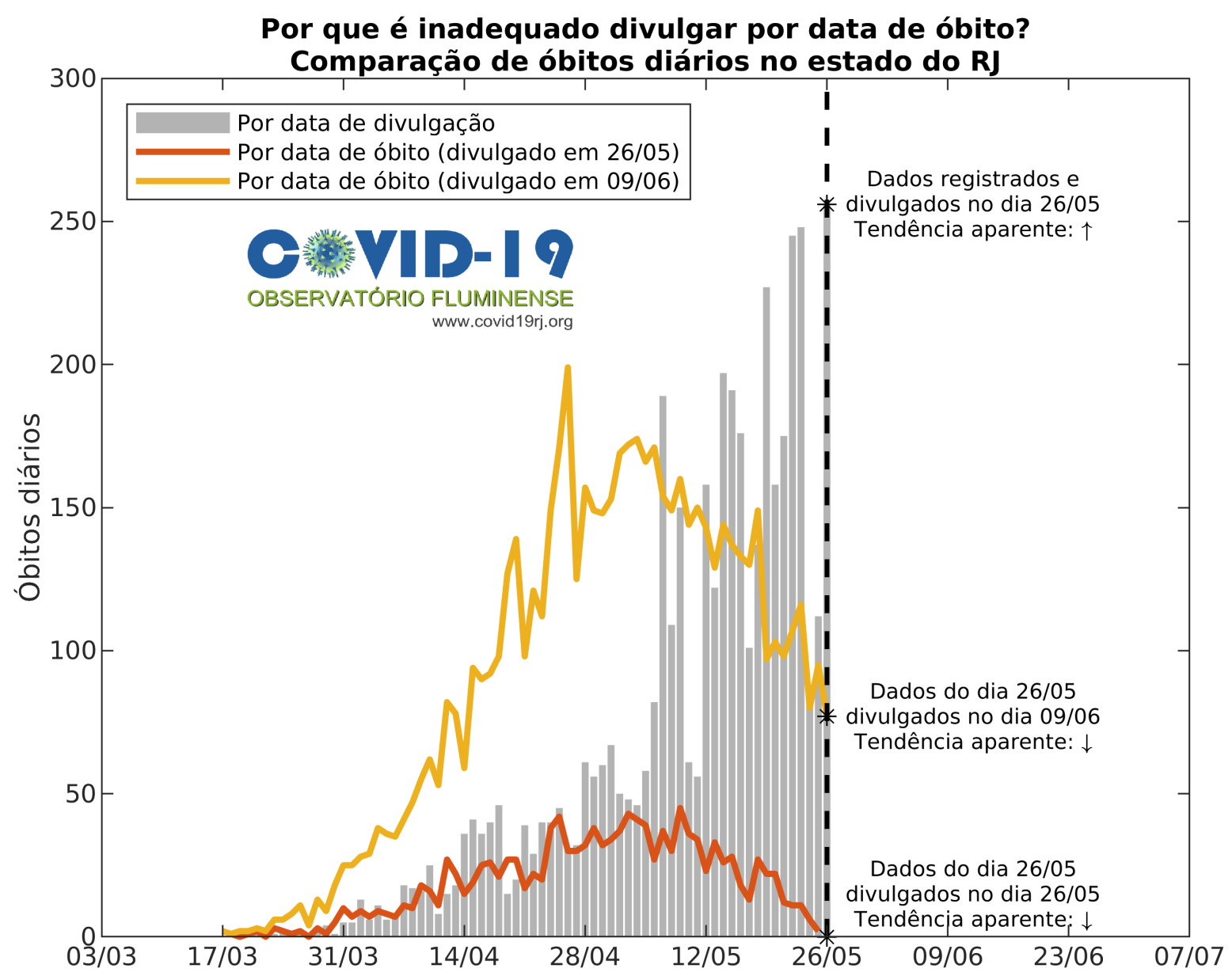

Figura 1: Comparação entre as diferentes formas de notificar os óbitos no Estado do Rio de Janeiro.

A divulgação dos dados por data de registro incorpora mortes de dias anteriores ao mesmo tempo que ignora mortes ocorridas no próprio dia, que somente serão notificadas em datas posteriores. Desta forma, há uma compensação. Considere que estamos num período estável da pandemia, isto é, quando a curva é quase plana. Considere ainda um comportamento estável no atraso de notificação, isto é, que a distribuição dos dias de atrasos na notificação de mortes se mantém ao longo do tempo. Neste caso, a curva de número de mortes por data de registro irá retratar muito bem a curva do número de mortes pela data de morte.

Por outro lado, na fase crescente, a curva por data de divulgação irá ter um atraso em relação à curva de data por data de óbito. Porém, a última tampouco pode ser construída durante a pandemia devido ao atraso na notificação das mortes. Assim, não há nenhum demérito em usar a curva de mortes por data de divulgação frente à curva de mortes por data de ocorrência nesse período, simplesmente por que a segunda também só pode ser construída após a primeira.

Por último, suponha uma fase de decréscimo real do número de mortes diárias. A curva por data de divulgação irá ter um pequeno atraso (de alguns dias) para refletir isso. Porém, quando o refletir o fará de forma inconteste. 
Sabe-se que o atraso nas notificações existe, desde o início das divulgações. Entendemos que a curva por data de divulgação acomoda de alguma forma essa limitação sistêmica. A curva por data de divulgação permite acompanhar a tendência do que ocorreu nos últimos dias de forma integrada. Complementarmente, se a divulgação usando a curva de óbitos diários apresenta sempre uma inflexão devido ao atraso no conhecimento das mortes, a curva por data de registro só decrescerá quando as mortes reduzirem consistentemente durante um período de tempo anterior. Assim, podemos dizer que a divulgação por data de registro (barras cinzas) é mais conservadora no retrato da pandemia, porém mais confiável na avaliação dos impactos da mesma.

O problema é ainda mais grave ao se divulgar apenas os óbitos ocorridos e registrados no dia, como foi cogitado pelo Ministério da Saúde. Se a divulgação fosse feita dessa forma, novos registros de mortes que ocorreram em dias anteriores à data de divulgação não seriam contabilizados. Esses dados entram no histórico da curva, pois ela sempre tem de ser atualizada e a simples divulgação do que ocorre no dia, não contempla todas as pessoas que perderam a vida pro COVID-19. A divulgação dos óbitos conforme eles foram registrados é mais justa inclusive com quem foi vítima da doença.

\section{Conclusão e recomendação}

À luz do exposto, vemos que a notificação por data de óbito é uma metodologia inadequada para monitorar a tendência da curva de mortes diárias decorrentes da COVID-19, sendo fortemente recomendado manter a divulgação dos óbitos por data de registro. A curva por data de óbito sempre apresentará uma tendência de queda na mortalidade e não será capaz de refletir o comportamento da epidemia devido ao desconhecimento de dados. Além disso, a curva por data de óbito é dinâmica (muda conforme a data) e sempre terá que ser atualizada para incorporar novas informações sobre mortes ocorridas em dias passados. Já a curva por data de registro não muda após a sua divulgação. Por ser estática, é muito mais segura para se avaliar uma tendência.

O mesmo ocorre quando se avaliam a confirmação de novos casos pela data de divulgação ou pela data de início dos sintomas. Para se avaliar a tendência do contágio da pandemia, devem-se utilizar os dados divulgados nos boletins diários e não as curvas por data de sintomas que também sofrem das mesmas fontes de atraso.

Vale ressaltar, que a divulgação dos dados por data de óbito ou início de sintomas é importante para se estudar o comportamento da pandemia. E, consequentemente, essas curvas devem ser divulgadas. Porém, essas informações só são úteis quando completas e devido aos atrasos nas notificações, sabe-se que em qualquer dia, o que elas refletem sobre o passado recente ainda não é um retrato confiável. Não há óbice à divulgação das curvas por data de óbitos juntamente com as curvas construídas por data de registro. Essa postura até forneceria mais informações à sociedade e aos pesquisadores que estão trabalhando no monitoramento da epidemia. Porém, as políticas de isolamento social devem ser feitas considerando a curva de dados registrados, pois esta é mais conservadora e representa melhor a tendência da curva de mortalidade e contágio causada pela pandemia naquele momento. 


\section{Referências}

[1] Secretaria Estadual de Saúde do Rio de Janeiro, 2020. http://painel.saude.rj.gov.br.

[2] A. Cunha Jr and et al. Relatório 01 Progresso da COVID-19 no Brasil e no Estado do Rio de Janeiro $21^{a}$ Semana Epidemiológica do Calendário 2020 (17/5/2020 até 23/5/2020). COVID-19: Observatório Fluminense, (24-05-2020). https://https://doi.org/10.12957/eduerj.covid19rj.relatorio1.

[3] A. Cunha Jr and et al. Relatório 02 Progresso da COVID-19 no Brasil e no Estado do Rio de Janeiro $22^{a}$ Semana Epidemiológica do Calendário 2020 (24/5/2020 até 30/5/2020). COVID-19: Observatório Fluminense, (31-05-2020). https://https://doi.org/10.12957/eduerj.covid19rj.relatorio2.

[4] A. Cunha Jr and et al. Relatório 03 Progresso da COVID-19 no Brasil e no Estado do Rio de Janeiro $23^{a}$ Semana Epidemiológica do Calendário 2020 (31/5/2020 até 06/6/2020). COVID-19: Observatório Fluminense, (07-06-2020). https://https://doi.org/10.12957/eduerj.covid19rj.relatorio3.

[5] A. Cunha Jr and et al. Relatório 04 Progresso da COVID-19 no Brasil e no Estado do Rio de Janeiro $24^{a}$ Semana Epidemiológica do Calendário 2020 (07/6/2020 até 13/6/2020). COVID-19: Observatório Fluminense, (14-06-2020). https://https://doi.org/10.12957/eduerj.covid19rj.relatorio4. 